\title{
PRES- and orthostatic-induced heart-rate changes as markers of labile hypertension: magnitude and reliability measures
}

\author{
Harald Rau*a, John J. Furedy ${ }^{\mathrm{b}}$, Thomas Elbert ${ }^{\mathrm{c}}$ \\ a Department of Medical Psychology, University of Tübingen, Tübingen, Germany \\ ${ }^{\mathrm{b}}$ Department of Psychology, University of Toronto, Toronto, Ontario, Canada \\ 'Department of Psychology, University of Konstanz, Konstanz, Germany
}

\begin{abstract}
Split-half and test-retest reliabilities of heart-rate responses to a baroreceptor manipulation and an orthostatic maneuver were compared between subjects with either normal or elevated blood-pressure. Ten subjects showing elevated resting blood-pressure and 11 normotensive subjects participated in two experimental sessions, each including heart-rate recordings during baroreceptor manipulation and orthostatic challenge. Carotid baroreceptors were manipulated by applying the baroreceptor-specific phase-related external suction (PRES) technique. The orthostatic stimulation procedure (OSP) was a change of body position from lying to standing. Heart rate responses evoked by OSP failed to discriminate significantly between the groups either in the magnitude or the (test/retest) reliability measure. The PRES procedure also failed to discriminate with the conventional magnitude measure, but the reliability measures showed significant differences. Paradoxically, the high-blood-pressure group manifested the higher baroreceptor reliability. The present findings are consistent with the view that operant conditioning produces phasic blood-pressure increases. In this view, blood-pressure increases activate the arterial baroreceptors which, in turn, dampen pain and/or stress sensitivity. Individuals showing high consistency (reliability) in their cardiovascular responses are more likely to learn this form of conditioning, and hence to eventually increase their tonic blood-pressure. High reliability of cardiovascular responses may therefore constitute a risk for hypertension. Aside from such theoretical considerations, the findings indicate that less conventional dependent variables like reliability may be worth exploring in the search for the etiology of essential hypertension, and that, in this search,
\end{abstract}

* Corresponding author, Institute for Medical Psychology and Behavioral Neurobiology, EberhardKarls University Tübingen, Gartenstrasse 29, D 72074 Tübingen, Germany. Tel.: (+ 49-7071) 294 222; Fax: (+49-7071) 295 956; E-mail: harald.rau@uni-tuebingen.de. 
specificity (relative to baroreceptor function) is more important than the magnitude of the heart-rate changes that are produced.

Keywords: Baroreceptor stimulation; Hypertension; PRES; Heart rate; Reliability

\section{Introduction}

Attempts to understand the factors involved in essential hypertension have included assessments of reactivity, as measured by phasic heart-rate changes to environmental changes (Fredrikson \& Matthews, 1990; Manuck et al., 1990; Pickering \& Gerin, 1990). One class of these manipulations has involved stimulation of the baroreceptors, which produces phasic bradycardia (Kirchheim, 1976). This assessment of reactivity may be particularly germane, because the baroreceptors regulate blood-pressure. There is accumulating evidence that baroreceptor sensitivity may be modulated in hypertensive subjects (Mancia et al., 1978, 1986; Sleight, 1991), and that this modified sensitivity may be a factor in the etiology of some forms of hypertension.

Baroreceptor stimulation methods include relatively global methods such as negative body tilting, which produces large-magnitude (over 30 beats $/ \mathrm{min}$ ) bradycardia (e.g. Furedy \& Poulos, 1976; Vaitl \& Gruppe, 1990; 1992). Pharmacological blood-pressure manipulation is another global method for stimulating baroreceptors, and determining their impact on the heart rate response (Mancia et al., 1977). However, it is unclear what other factors, beside baroreceptor regulation, produce these heart rate effects. At the other end of the global-specific continuum is the recently developed Phase-Related-External-Suction (PRES) technique, which, though still non-invasive, produces more specific stimulation of the carotid baroreceptors. The most recently developed form of PRES (Rau et al., 1992) is maximally specific in the sense that psychological or other non-specific (relative to baroreceptor stimulation) factors can be ruled out, since the stimulation and control conditions cannot be differentiated by most subjects (Furedy et al., 1992). However, the bradycardic change induced by PRES is only some 3 beats $/ \mathrm{min}$, and hence could be considered by some theorists to be 'biologically insignificant' (Obrist, 1976). Also, the procedure is artificial, and hence may not represent naturally occurring events that are relevant to blood-pressure regulation.

An orthostatic stimulation procedure (OSP) which involves changing from a supine to a standing posture is a commonly used procedure in medical practice. It is employed to examine the cardiovascular regulation in response to venous pooling of blood in the lower body parts. The OSP produces larger changes in heart rate (tachycardia of over 10 beats/min) than PRES, and is also less artificial and hence potentially more 'ecologically valid'.

Nevertheless, it is an empirical question whether these aspects make OSP superior to PRES in differentiating between subjects with high and low tonic blood-pressure levels. Indeed, the relevant dependent variable may not be response magnitude at all, but the stability or reliability of the evoked change in heart rate which prevents the organism from producing frequent hypertonic phases. 
Accordingly, the present study contrasted PRES-induced bradycardic responses with OSP-induced tachycardic responses. In addition to the conventionally-assessed response-magnitude dependent variable, reliability was also assessed in a test/retest format for both PRES and OSP (sessions separated by several weeks), and in a split-half format for PRES. Tonic blood-pressure was varied between groups by selecting subjects with either normal or above-normal blood pressure levels. This allowed assessment of the extent to which these reactivity measures could differentiate between the differing tonic blood-pressure groups.

\section{Methods}

\subsection{Subjects}

In order to select subjects with slightly elevated blood-pressure (here labeled as 'high blood-pressure') and subjects with normal blood-pressure ('normal bloodpressure'), blood-pressure of about 200 students of the University of Tübingen was taken using an automatic arm cuff device (BOSO digimat) which detects Korotkov sounds during deflation of the cuff. Subjects showing a diastolic blood-pressure below $85 \mathrm{mmHg}$ or above $95 \mathrm{mmHg}$ in at least two independent measurements were invited to visit the laboratory of the department for Medical Psychology during the following week. Subjects reporting cardiovascular, neurological, psychiatric or other serious diseases or being on chronic or actual drug regimen were excluded. All subjects who have been asked to participate agreed and performed the experimental tasks which were approved by the local ethics committee. This procedure resulted in a sample of 11 normotensive subjects and 10 subjects with elevated blood-pressure. They received $40 \mathrm{DM}$ for participation in this session. Characteristics of and significant differences between both groups are outlined in Table 1.

Aside from all blood-pressure variables, there were no significant differences between groups.

Four to 8 weeks after the first experimental session, 10 normotensive and nine subjects of the high blood-pressure group participated in the second session which had the identical arrangement as the first one. These subjects again received $40 \mathrm{DM}$ for participation.

\subsection{Baroreceptor manipulation}

\subsubsection{Orthostatic stimulation procedure}

The first part of each experimental session consisted of the orthostatic test. Subjects were in a standing position for $2 \mathrm{~min}$. During this interval, blood-pressure and heart-rate were measured once using an automatic blood-pressure monitor (BOSO digimat) which is based on the detection of Korotkov sounds during deflation of the arm cuff. Then, subjects were asked to take a supine position for 3 min. Blood-pressure and heart rate were measured during the beginning and at the end of this period. During the following $2 \mathrm{~min}$, subjects had to take an upright standing position. Blood-pressure and heart rate were again measured at the end of 
this interval. For the analysis reported in this paper, the heart rate response to the change in body position from supine to standing posture was calculated as a difference score (arithmetic difference between both positions). Heart rate recordings during the OSP were based on the Korotkov sounds automatically detected during deflation of the arm cuff.

\subsubsection{PRES technique}

The arterial baroreceptors of the carotid sinus were manipulated using a neck cuff (as described by Eckberg et al., 1975). The neck cuff was connected to a device consisting of a motor (from an ordinary vacuum cleaner) and four valves (the device and the whole technique is described in detail by Rau et al., 1992). This arrangement allows rapid changes of air pressure to occur within the neck cuff. The baroreceptors are stretch receptors responding maximally to rapid stretching of blood vessels. By applying short (e.g. $250 \mathrm{~ms}$ ) suction bursts during systole, the systolic stretching is amplified. A subsequent blowing burst of equal length tends to constrict the vessels during the diastole. A train of multiple systolic suction and diastolic blowing bursts leads to increased variation of stretching of the carotid

Table 1

Characteristics and significant differences between groups

\begin{tabular}{|c|c|c|c|}
\hline Characteristic & All subjects & $\begin{array}{l}\text { Low } \\
\text { blood-pressure }\end{array}$ & $\begin{array}{l}\text { High } \\
\text { blood-pressure }\end{array}$ \\
\hline Age (years) & $27.8(7.3)$ & $25.7(8.1)$ & $30.0(5.8)$ \\
\hline Weight (kg) & $73.7(10.9)$ & $72.0(8.1)$ & $75.8(13.8)$ \\
\hline Nicotine comsumption (cigarettes/day) & $5.0(8.4)$ & $3.7(8.1)$ & $6.6(9.0)$ \\
\hline Physical activity (sports, min/week) & $147.4(169.2)$ & $172.0(132.1)$ & $120.0(207.9)$ \\
\hline $\begin{array}{l}\text { First diastolic blood-pressure during } \\
\text { session } 1(\mathrm{mmHg})^{*}\end{array}$ & $89.33(16.0)$ & $78.0(9.4)$ & $101.8(11.9)$ \\
\hline $\begin{array}{l}\text { First systolic blood-pressure during } \\
\text { session } 1(\mathrm{mmHg})^{*}\end{array}$ & $134.0(12.8)$ & $125.8(9.7)$ & $143.0(9.3)$ \\
\hline $\begin{array}{l}\text { Mean diastolic blood-pressure during } \\
\text { session } 1(\mathrm{mmHg})^{*}\end{array}$ & $87.6(15.5)$ & $76.2(5.4)$ & $100.1(13.0)$ \\
\hline $\begin{array}{l}\text { Mean systolic blood-pressure during } \\
\text { session } 1(\mathrm{mmHg})^{*}\end{array}$ & $134.5(16.1)$ & $123.0(11.6)$ & $147.1(9.6)$ \\
\hline $\begin{array}{l}\text { First diastolic blood-pressure during } \\
\text { session } 2(\mathrm{mmHg})^{*}\end{array}$ & $88.1(12.8)$ & $79.0(6.6)$ & $98.2(10.2)$ \\
\hline $\begin{array}{l}\text { First systolic blood-pressure during } \\
\text { session } 2(\mathrm{mmHg})^{*}\end{array}$ & $132.5(15.6)$ & $123.8(13.9)$ & $142.1(11.3)$ \\
\hline $\begin{array}{l}\text { Mean diastolic blood-pressure during } \\
\text { session } 2(\mathrm{mmHg})^{*}\end{array}$ & $86.3(12.9)$ & $76.6(4.9)$ & $97.1(10.0)$ \\
\hline $\begin{array}{l}\text { Mean systolic blood-pressure during } \\
\text { session } 2(\mathrm{mmHg})^{*}\end{array}$ & $129.9(16.1)$ & $120.0(12.0)$ & $141.0(12.6)$ \\
\hline
\end{tabular}

*Significant group differences at $P<0.001$.

**Significant group differences at $P<0.0001$.

First blood-pressure measurements were done before the experiment began and before instructions were given. During each session, 5 blood-pressure records were taken; the average of these measurements are reported here as 'mean blood-pressure'. 
sinus ('stimulation condition'). The baroreceptors respond to this increased variation and increase their activation. For applying a control condition, a blowing burst is presented during systole, tending to counteract the systolic dilation. During diastole, a suction burst of equal length is applied to the neck, tending to dampen the diastolic vasoconstriction. As a result, the variance in stretching is reduced under this condition ('control condition'). Accordingly, the carotid baroreceptors are less activated during this control condition. Most subjects are unaware of the relation of the external suction and blowing bursts to their own heart cycle, and of the actual condition (stimulation vs. control). As shown by Furedy et al. (1992), most subjects are unable to discriminate between the two conditions. For triggering the pressure bursts, an electrocardiogram was recorded from non-standardized electrode positions producing large $\mathbf{R}$-waves. The signal was amplified using a Beckman amplifier. The amplified signal was fed into a modified Beckman cardiotachometer module which generated a TTL output of 200 ms duration each time an R-wave was detected. This TTL signal was fed into the digital input of an MS-DOS compatible computer which was programmed using the ASYST language. To apply a stimulation trial, the computer initiated a suction burst $100 \mathrm{~ms}$ after an $\mathrm{R}$-wave was detected. This suction burst lasted for the duration which was calculated as half of the mean inter beat intervals of the earlier trials minus $100 \mathrm{~ms}$. After this period, a positive pressure was applied for the same duration of time. To the beginning of the next suction burst, neutral cuff pressure was generated. Then, the same pressure sequence began again, until the end of the manipulation period was achieved. For the control trials, the positive and negative pressure bursts were simply reversed. The time of each R-wave occurrence was detected with an accuracy of $1 \mathrm{~ms}$ and stored on the hard disk of the computer. Off line, the inter-beat interval was calculated and converted to heart rate (beats/min). Heart rate responses to both conditions were calculated as mean heart rate during seconds $2-6$ of the manipulation interval. Baseline heart-rate ( $1 \mathrm{~s}$ before the beginning of the manipulation period) was subtracted from this score. The resulting value was expressed as the heart rate response score, with positive values and negative values indicating, respectively, heart rate acceleration and deceleration during the manipulation period.

In the present experiment, 24 PRES-stimulation and 24 control trials were presented in an individually pseudo-randomized sequence to each subject. Each trial included a baroreceptor manipulation interval of $6 \mathrm{~s}$ duration, a 1-s baseline and a 1 -s post-baseline period. The intertrial interval varied randomly between 15 and 25 s. Heart-rate responses were off-line averaged over all trials, separated for both conditions and the subjects.

\subsection{Statistical analyses}

In order to evaluate the magnitude measures, the heart rate changes to the orthostatic-stimulation procedure and the PRES technique were entered into a $2 \times$ 2 ANOVA with 'group' (normotensives vs. labile hypertensives) and 'procedure' (OSP vs. PRES), respectively, as the between- and within-subject factors. For the PRES technique, heart rate differentiation between stimulation and control trials was used, controlling for non-specific heart rate responses. Because heart rate should decelerate in response to the PRES stimulation of the baroreceptors, but 


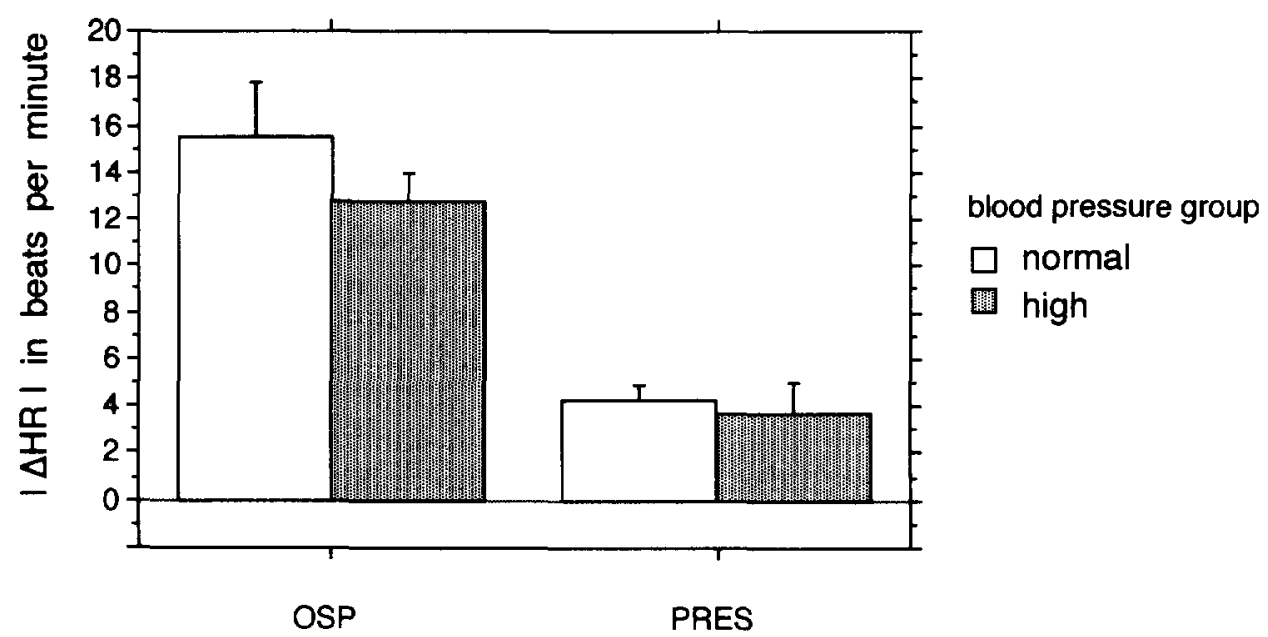

Fig. 1. Absolute heart rate changes (beats/min) in response to OSP (orthostatic stimulation procedure) and PRES (phase-related external suction) technique, separated for the two blood-pressure groups. Absolute values are used for this presentation and analysis in order to compare heart rate responses between both manipulations.

accelerate in response to the baroreceptor unloading of the OSP, absolute values were calculated for all measures.

For the reliability analyses, heart rate scores were subjected to correlational analyses. In order to compare correlations between groups, their values were converted to Fisher's $z$-values which can be compared in terms of whether they differ statistically. For measuring the test/retest reliability, heart rate response scores of session 1 were correlated with the equivalent scores of session 2 , for the OSP, the PRES-stimulation and the PRES-control trials. Split-half reliability for the PRES-induced heart rate responses was calculated by performing a correlation between the mean heart rate responses to the first 24 trials (12 stimulation and 12 control trials) and the second 24 trials (again 12 stimulation and 12 control trials).

\section{Results}

\subsection{Magnitude measures}

Fig. 1 shows absolute delta change scores (bradycardia and tachycardia for PRES and OSP, respectively) as a function of Manipulation Type (PRES versus OSP) and Tonic Blood-pressure (normal vs.high blood-pressure). As suggested by the figure, a $2 \times 2$ ANOVA with Manipulation Type and Tonic Blood-pressure as factors yielded only a significant main effect for Manipulation Type $(F(1,17)=57.872 ; P<0.0001)$ with larger changes in the OSP $(14.2$ beats $/ \mathrm{min})$ than in the PRES $(4.0$ beats/min) conditions. The other main effect $(F(1,17)=0.909)$ and interaction effect $(F(1,17)=0.805)$ were not significant.

\subsection{Reliability measures (test/retest)}

The larger-magnitude OSP-induced response showed no significant test/retest reliability. The correlation values were 0.051 for the two groups combined $(n=19)$, 
0.069 for the normotensive group $(n=10)$, and 0.324 for the labile hypertensive group $(n=9)$. For all these values, $p>0.05$, although it will be noted that the highest (but not significant) value emerged in the high blood-pressure group.

The lower-magnitude PRES-induced response showed a much more significant and orderly pattern of test/retest correlations, and Fig. 2 provides scattergrams in addition to values for these correlations. As this figure indicates, the PRES-stimulation responses showed high reliability $(r=0.717)$ for the two groups combined (top left panel), but the high blood-pressure group (top right panel) accounted for all of this effect, since the normotensive group's correlation value of $r=0.269 \mathrm{did}$ not yield statistical significance, in contrast to the high blood-pressure group's unusually high value of $r=0.929$. This indicates a qualitative difference between the groups with respect to test/retest reliability of PRES-induced responding. As Fig. 2 indicates, there was only one subject in the high blood-pressure group who had an extraordinarily strong response to the PRES-stimulation condition. Removing this outlying subject from the analysis resulted in a still highly significant correlation between heart rate changes of both sessions $(r=0.851, P<0.005)$ in the high blood-pressure group. This single subject therefore was not responsible for the qualitative difference between both groups.

The PRES-control response reliabilities are shown in the bottom panels of Fig. 2 . As for the OSP-induced responses, no significant test/retest reliability values emerged from these data.

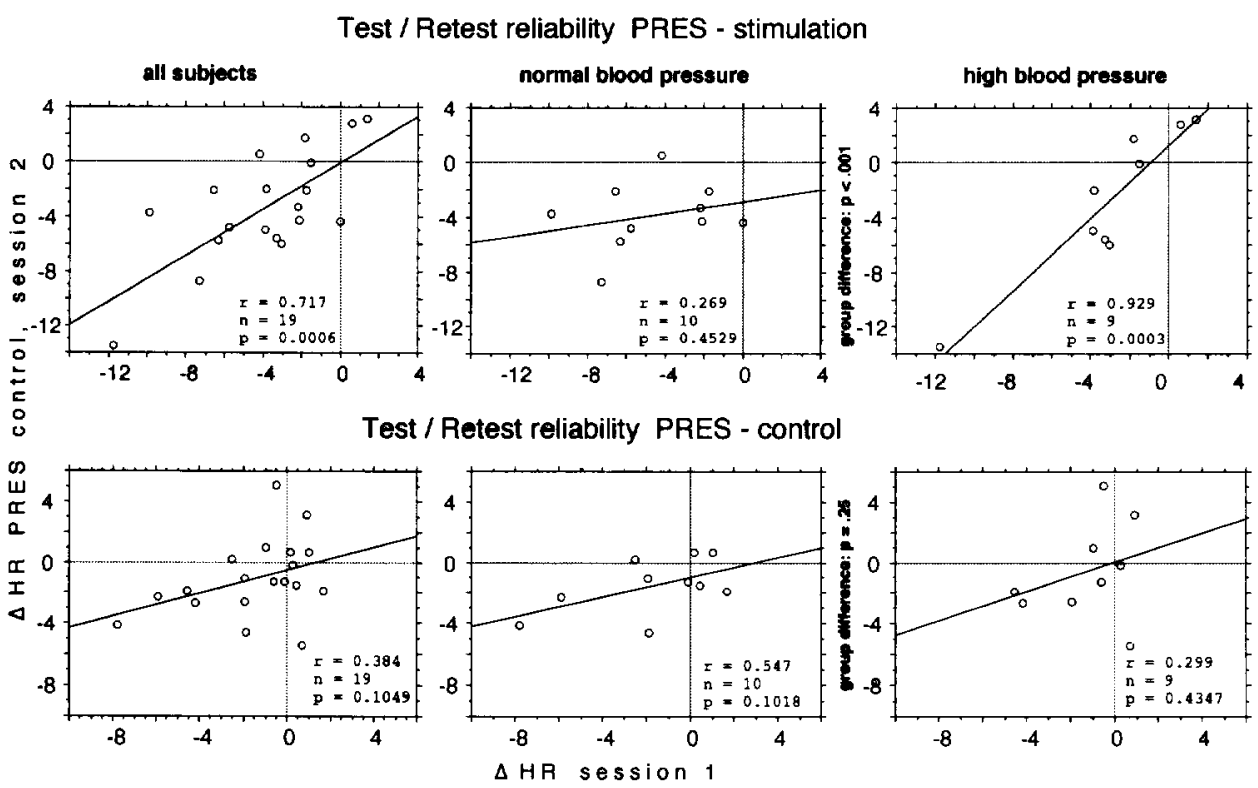

Fig. 2. Test/retest reliability of PRES-stimulation induced (upper panel) and PRES-control induced (lower panel) heart-rate changes. 
Split half reliability PRES - stimulation

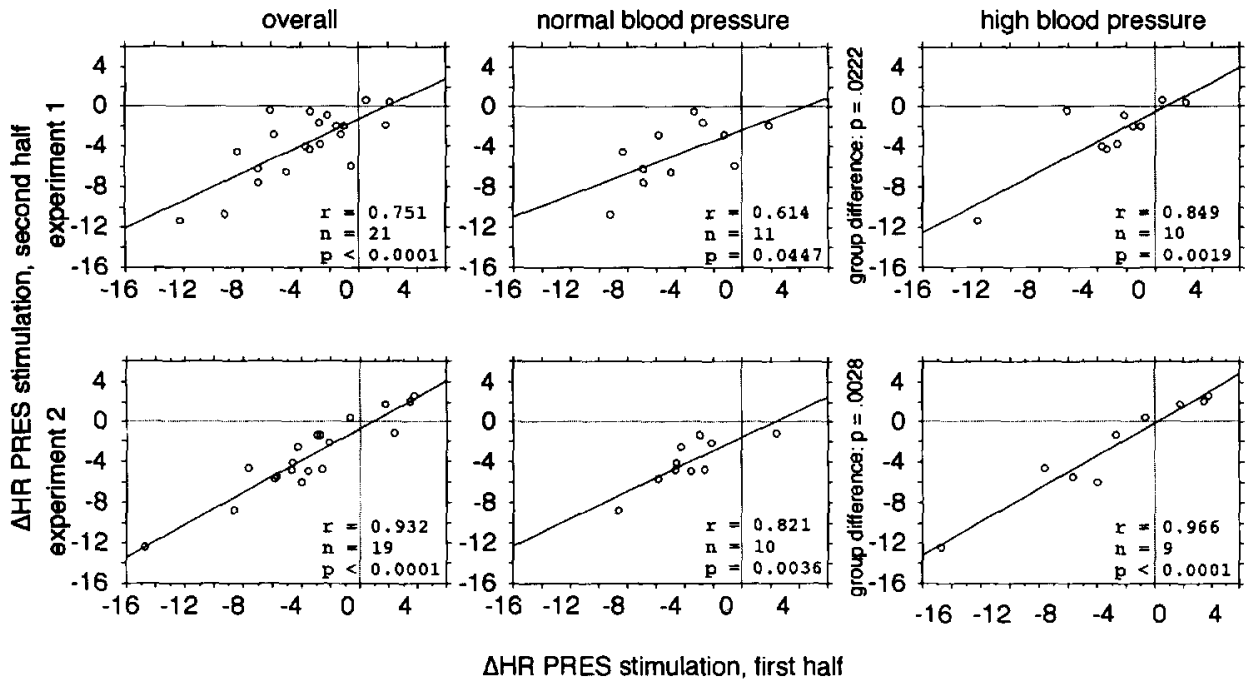

Split half reliability PRES - control
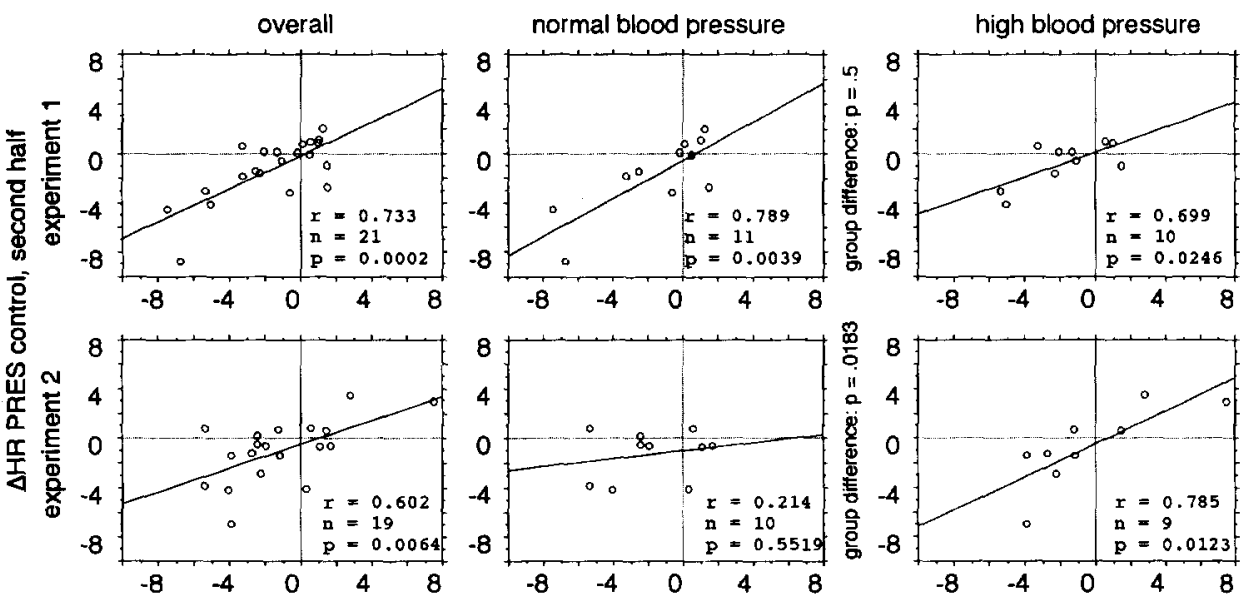

$\triangle$ HR PRES control, first half

Fig. 3. Split-half reliabilities of PRES-stimulation (upper part) and PRES-control (lower part) induced heart rate changes.

\subsection{Reliability measures (split-half) for PRES}

These values were obtained by splitting each experiment into first and second 24-trial halves with data from 21 subjects being available for this analysis for session 1 and only 19 of those subjects for session 2. Fig. 3 shows the results for the 
PRES-stimulation (upper part) and PRES-control (lower part) responses. During both sessions, all correlations were significant, but the group difference did emerge in the quantitative sense that the reliabilities were significantly higher in the high blood-pressure group than in the normotensives. Noteworthy also is the relatively high absolute values of the correlations in Fig. 3.

The PRES-control split-half reliabilities are shown in the bottom part of Fig. 3. In contrast to the test/retest results, the split-half combined-group values were significant, though not as high as those found in corresponding PRES-stimulation values. Also, in session 2 (though not in session 1, where the non-significant trend is in the opposite direction), the reliability values in the high blood-pressure group significantly exceeded those of the normotensives, $P<0.05$.

\section{Discussion}

Results based on the conventionally-used magnitude dependent variable indicated that neither the OSP-induced tachycardia nor the PRES-induced bradycardia could differentiate between the groups that differed in tonic blood-pressure. In contrast, the reliability-based dependent variable for PRES-induced tachycardia produced clear differentiation, which was even of the qualitative sort in the case of the test/retest correlations. To our knowledge this is the first time that reliability of a challenge-induced bradycardia has been identified as a marker for hypertension. In physiological terms this may be important for the area concerned with the psychophysiological factor in diseases (psychosomatic medicine), where the question of response specificity has been an important one (Stern \& Sison, 1990). The phenomenon that some subjects tend to respond to a variety of external or internal cues with one physiological system (here: the cardiovascular system) might increase the vulnerability of this system (Manuck et al., 1990). The present result yielded much higher reliability in subjects (high blood-pressure group) who might have already increased risk for development of hypertension.

The relative reliability outcomes between the OSP and PRES procedures need to be interpreted with the caveat that the comparison was between two common forms of each procedure, which, in fact, differ as regards statistical and measurement properties. Specifically, in standard practice the OSP is a single-trial procedure in which heart rate is calculated on the basis of a few Korotkov sounds, whereas the PRES procedure employs multiple trials and (the more accurate) method of calculating heart rate on the basis of many clearly detectable R-waves of the EKG. These statistical and measurement feature differences tend to favor the PRES procedure in terms of potential reliability, so no conclusion regarding the relative efficacy of 'ideal' forms of the two procedures is possible on the basis of our results. Such a conclusion would require examination of a multiple-trials, R-wave-based form of the PRES procedure. What is clear is that the common form of the PRES procedure is superior to the common form of the OSP as a risk indicator for the development of hypertension.

It may seem paradoxical that more reliable responding of the baroreceptors is related to a risk of development of hypertension. However, the theory by Dworkin 
$(1988,1991$; Dworkin et al., 1979) offers a possible hypothetical mechanism that would account for this counter-intuitive link. The theory asserts that naturally occurring blood-pressure increases can lead to dampening of pain and aversive experience. This dampening seems to be mediated through the arterial baroreceptors (Droste et al., 1994; Kardos et al., 1994; Rau et al., 1993a,b, 1994), especially in hypertensive or labile hypertensive subjects (Elbert et al., 1988). The organism's experience of this pain-inhibiting effect of blood-pressure-induced baroreceptor activation may negatively reinforce the initiation of phasic blood-pressure increases. These temporary phases ('lability') of increased blood-pressure constitute a risk for the development of manifest hypertension (Pickering \& Gerin, 1990). This theory, which has been recently confirmed in a longitudinal investigation (Elbert et al., 1994), could explain the link found in the present data: Subjects responding with a reliable activation of their baroreceptors may perceive the reinforcing quality of blood-pressure increases, and may use this effect as a coping strategy. As a side effect, labile hypertension will develop.

However, independent of such theoretical speculations about the mechanisms that may lie behind the connection between PRES-induced bradycardia reliability and hypertension, the present findings do suggest that the larger (and more 'natural') responses may be less useful in reactivity studies of hypertension than smaller (and more artificially-induced) responses, even if these responses are, in themselves, too small to be of any metabolic significance. Moreover, the fact that the magnitude dependent variable itself failed to differentiate between the two tonic-blood-pressure groups, whereas the reliability dependent variable did so clearly in the case of PRES-induced bradycardia, suggests that less conventional dependent variables may be worth exploring in the search for the etiology of essential hypertension.

\section{Acknowledgment}

This research was supported by the Deutsche Forschungsgemeinschaft (El101/33). We also acknowledge the assistance by Beate Sauter and Matthias Vöhringer in data collection.

\section{References}

Droste, C., Kardos, A., Brody, S., Greenlee, M.W., Roskamm, H. \& Rau, H. (1994). Baroreceptor stimulation: Pain perception and sensory thresholds. Biological Psychology, 37, 101-113.

Dworkin, B. (1988). Hypertension as a learned response: The baroreceptor reinforcement hypothesis. In T. Elbert, W. Langosch, A. Steptoe and D. Vaitl (Eds.), Behavioral medicine in cardiovascular disorders (pp. 17-47). Chichester: John Wiley \& Son.

Dworkin, B. (1991). The baroreceptor reinforcement instrumental learning (BR-IL) model of essential hypertension: biological data, quantitative mechanisms, and computer modeling. In A.P. Shapiro and U. Baum (Eds.), Behavioral aspects of cardiovascular disease (pp. 213-245). Hillsdale, NJ: Lawrence Erlabaum Assosiates, Publishers.

Dworkin, B., Filewich, R.J., Miller, N.E., Craigmyle, N. \& Pickering, T.G. (1979). Baroreceptor activation reduces reactivity to noxious stimulation: implications for hypertension. Science, 205, $1299-1301$. 
Eckberg, D.L., Cavanaugh, M.S., Mark, A.L. \& Abboud, F.M. (1975). A simplified neck suction device for activation of carotid baroreceptors. Journal of Laboratory and Clinical Medicine, 85, 167-- 173.

Elbert, T., Dworkin, B.R., Rau, H., Pauli, P., Birbaumer, N., Droste, C. \& Brunia, C.H.M. (1994). Sensory effects of baroreceptor activation and perceived stress together predict long-term blood pressure elevations. International Journal of Behavioral Medicine, 1(3), 215-228.

Elbert, T., Lutzenberger, W., Rockstroh, B., Kessler, M., Pietrowsky, R. \& Birbaumer, N. (1988). Baroreceptor stimulation increases pain threshold in borderline hypertensives. Psychophysiology, 25 , $25-29$.

Fredrikson, M. \& Matthews, K.A. (1990). Cardiovascular responses to behavioral stress and hypertension: a meta-analytic review. Annals of Behavioral Medicine, 12, 30-39.

Furedy, J., Rau, H. \& Roberts, L. (1992). Physiological and psychological differentiation of bidirectional baroreceptor carotid manipulation in humans. Physiology and Behavior, 52, 953-958.

Furedy, J.J. \& Poulos, C.X. (1976). Heart-rate decelerative Pavlovian conditioning with tilts as UCS: Towoards behavioral control of cardiac dysfunction. Biological Psychology, 4, 93-106.

Kardos, A., Rau, H., Greenlee, M.W., Droste, C., Brody, S. \& Roskamm, H. (1994). Reduced pain during baroreceptor stimulation in patients with symptomatic and silent myocardial ischemia. Cardiovascular Research, 28, 515-518.

Kirchheim, H.R. (1976). Systemic arterial baroreceptor reflexes. Physiological Reviews, 56, 100-176.

Mancia, G., Ferrari, A., Gregorini, L., Valentini, R., Ludbrook, J. \& Zanchetti, A. (1977). Circulatory reflexes from carotid and extracarotid baroreceptor areas in man. Circulation research, 41, 309-315.

Mancia, G., Ferrari, A.U. \& Zanchetti, A. (1986). Reflex control of the circulation in experimental and human hypertension. In A. Zanchetti and R.C. Tarazi (Eds.), Handbook of Hypertension (pp. 47-68). Amsterdam: Elsevier Science Publishers BV.

Mancia, G., Ludbrook, J., Ferrari, A., Gregorini, L. \& Zanchetti, A. (1978). Baroreceptor reflexes in human hypertension. Circulation Research, 43, 170-177.

Manuck, S.B., Kasprowicz, A.L. \& Muldoon, M.F. (1990). Behaviorally-evoked cardiovascular reactivity and hypertension: Conceptual issues and potential associations. Annals of Behavioral Medicine, 12 , $17-29$.

Obrist, P.A. (1976). The cardiovascular behavioral interaction - as it appears today. Psychophysiology, $13,95-107$.

Pickering, T.G. \& Gerin, W. (1990). Cardiovascular reactiv ity in the laboratory and the role of behavioral factors in hypertension: A critical review. Annals of Behavioral Medicine, 12, 3-16.

Rau, H., Elbert, T., Geiger, B. \& Lutzenberger, W. (1992). PRES: The controlled noninvasive stimulation of the carotid baroreceptors in humans. Psychophysiology, 29, 165-172.

Rau, H., Brody, S., Brunia, C.H.M., Damen, E.P.J. \& Elbert, T. (1993a). Activation of carotid baroreceptors inhibits spinal reflexes in man. Electroencephalography and Clinical Neurophysiology, 89 , $328-334$.

Rau, H., Pauli, P., Brody, S., Elbert, T. \& Birbaumer, N. (1993b). Baroreceptor stimulation alters cortical activity. Psychophysiology, 30, 322-325.

Rau, H., Brody, S., Larbig, W., Pauli, P., Vöhringer, M., Harsch, B., Kröling, P. \& Birbaumer, N. (1994). The effects of PRES baroreceptor stimulation on thermal and mechanical pain threshold in borderline hypertensives and normotensives. Psychophysiology, 31, 480-485.

Sleight, P. (1991). Baroreceptors and hypertension. In P.B. Persson and H.R. Kirchheim (Eds.), Baroreceptor reflexes. Integrative functions and clinical aspects (pp. 271-292). Berlin: Springer-Verlag.

Stern, R.M. \& Sison, C.E.E. (1990). Response patterning. In J.T. Cacioppo and L.G. Tassinary (Eds.), Principles of psychophysiology. Physical, social, and inferential elements (pp. 193-215). Cambridge: Cambridge University Press.

Vaitl, D. \& Gruppe, H. (1990). Changes in hemodynamics modulate electrical brain activity. Journal of Psychophysiology, 4, 41-49.

Vaitl, D. \& Gruppe, H. (1992). Body position and changes in EEG. Journal of Psychophysiology, 6 , 111-118. 